\title{
EFFECTIVE DETERMINATION OF THE DECOMPOSITION OF THE RATIONAL PRIMES IN A CUBIC FIELD
}

\author{
PASCUAL LLORENTE AND ENRIC NART
}

\begin{abstract}
The decomposition of the rational primes in a cubic field $K$ is determined in terms of the coefficients of a defining polynomial of $K$. As a consequence, the discriminant $D$ of $K$ is straightforwardly computed and the cubic fields with index $i(K)=2$ are easily characterized.
\end{abstract}

Introduction. The study of the decomposition of the rational primes in a cubic field $K$ is well known if $K$ is cyclic $[3, \S 24]$ and the noncyclic case was developed by Hasse [5] and more recently by Martinet and Payan [6]. However, these results are not sufficient to determine such decompositions in terms of a defining polynomial of $K$. This is the problem we solve in this paper (Theorem 1). As a consequence, we obtain a straightforward computation of the discriminant of $K$ (Theorem 2) and we reobtain in a completely elementary way a well-known theorem of Hasse [5, Theorem 6] on the possible values of the discriminant of a cubic field (Theorem 3). Finally, as another consequence of Theorem 1, we find a characterization of the cubic fields in which 2 is a common index divisor, which improves the given by Tornheim in [8, Theorem 2] (Theorem 4).

Most of our results are easily extendible to the case of relative cubic extensions, but we will not make these generalizations explicit.

Let $K$ be a cubic field. We can suppose that $K=\mathbf{Q}(\theta)$, where $\theta$ is a root of an irreducible polynomial of the type

$$
f(X)=X^{3}-a X+b, \quad a, b \in \mathbf{Z} .
$$

The discriminant of $f(X)$ is $\Delta=4 a^{3}-27 b^{2}$ and if we denote by $D$ the discriminant of $K$ we have $\Delta=i(\theta)^{2} \cdot D$, where $i(\theta)$ denotes the index of $\theta$.

For every prime $p \in \mathbf{Z}$ and integer $m \in \mathbf{Z}$ we denote by $v_{p}(m)$ the greatest exponent $k$ such that $p^{k} \mid m$. If for any prime $p$ we have

$$
v_{p}(a) \geqslant 2 \text { and } v_{p}(b) \geqslant 3 \text {, }
$$

then $\theta / p$ is an algebraic integer whose equation is $X^{3}-\left(a / p^{2}\right) X+\left(b / p^{3}\right)$. Therefore, we can assume that (1) is not satisfied for any prime $p$.

Let $s_{p}=v_{p}(\Delta)$ and $\Delta_{p}=\Delta / p^{s_{p}}$ for every prime $p$.

Received by the editors May 21, 1981

1980 Mathematics Subject Classification. Primary 12A30.

Key words and phrases. Cubic field, ramification, discriminant, index of a number field.

(C) 1983 American Mathematical Society $0002-9939 / 82 / 0000-0768 / \$ 02.25$ 
THEOREM 1. The primes of $\mathbf{Q}$ decompose in $K$ as follows:

Decomposition of 2.

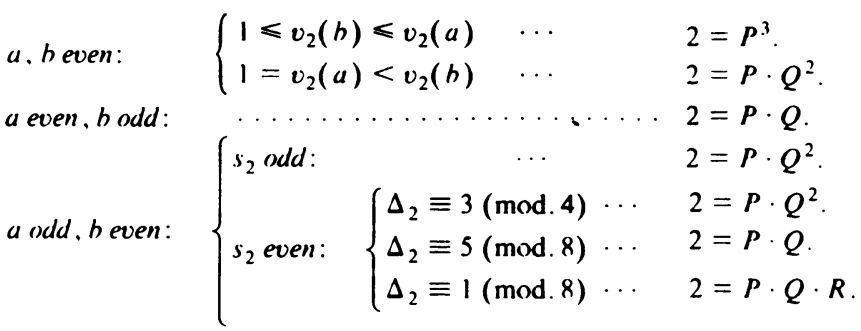

a. $b$ odd:

$2=P$.

Decomposition of 3 .

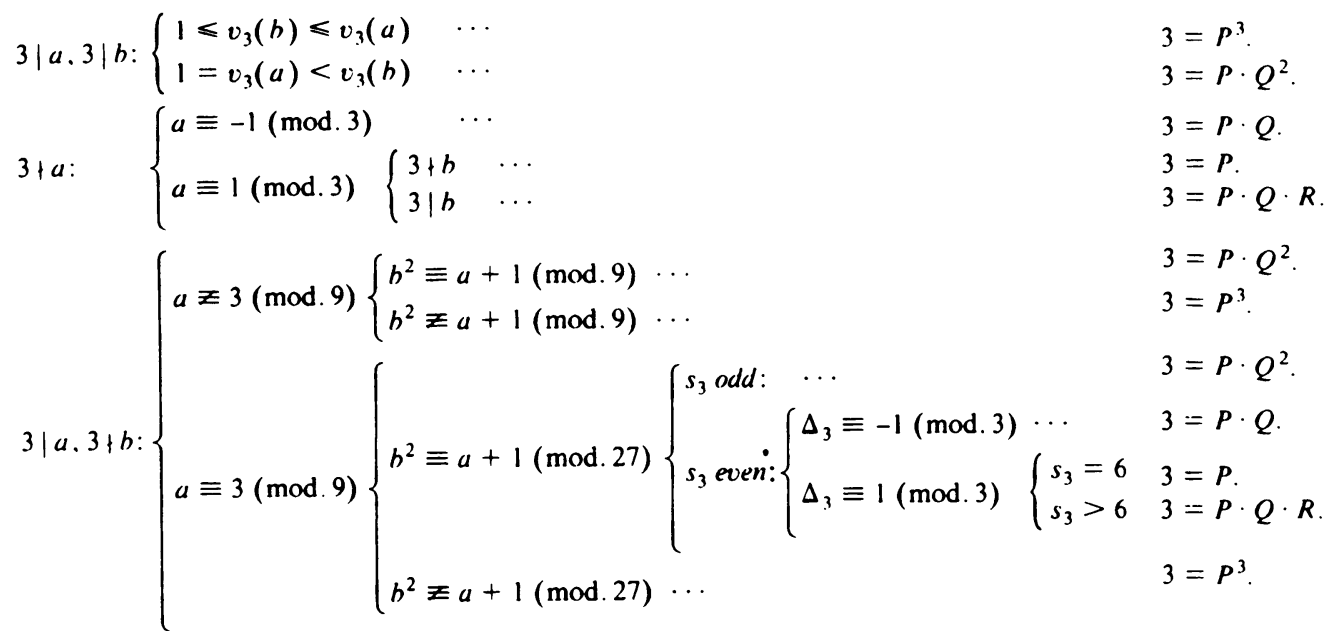

Decomposition of $p>3$.

$$
\begin{aligned}
& p|a, p| b: \quad\left\{\begin{array}{l}
1 \leqslant v_{p}(b) \leqslant v_{p}(a) \\
1=v_{p}(a)<v_{p}(b)
\end{array}\right. \\
& p=P^{3} \text {. } \\
& p \mid a, p \nmid b: \quad\left\{\begin{array}{l}
p \equiv-1(\bmod 3) \\
p \equiv 1(\bmod 3)\left\{\begin{array}{l}
(b / p)_{3}=1 \\
(b / p)_{3} \neq 1
\end{array}\right.
\end{array}\right. \\
& p=P \cdot Q^{2} \text {. } \\
& p \nmid a, p \mid b: \quad\left\{\begin{array}{l}
(a / p)=1 \\
(a / p)=-1
\end{array}\right. \\
& p=P \cdot Q \text {. } \\
& p=P \cdot Q \cdot R \text {. } \\
& p=P \text {. }
\end{aligned}
$$

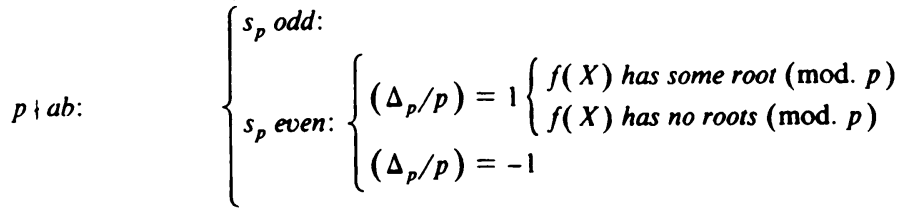

$$
\begin{aligned}
& p=P \cdot Q \cdot R . \\
& p=P \cdot Q \text {. } \\
& p=P \cdot Q^{2} \text {. } \\
& p=P \cdot Q \cdot R \text {. } \\
& p=P \text {. } \\
& p=P \cdot Q \text {. }
\end{aligned}
$$


REMARK. This theorem is particularly interesting for $p=2$ and 3 ; not only because the exceptional behaviour of these primes makes its study more difficult, but specially because it solves these cases in a completely effective way. For $p>3$ effectiveness depends on checking if an integer is a quadratic or cubic residue $(\bmod . p)$ and on checking if the polynomial has roots (mod. $p$ ). Nevertheless, we note that for small primes effectiveness is also complete since the quadratic and cubic residues can be easily computed and because we have found an interesting method, to be developed in a later paper, to determine whether $f(X)$ has roots (mod. $p$ ) or not. Thus, if $p \nmid a b, s_{p}$ even and $\left(\Delta_{p} / p\right)=1$ we have found, for instance, that

$$
\begin{array}{ll}
5=P \cdot Q \cdot R . \Leftrightarrow a^{3} \equiv 3 b^{2} & (\bmod .5) \\
7=P \cdot Q \cdot R . \Leftrightarrow a^{3} \equiv 5 b^{2} & (\bmod .7) \\
11=P \cdot Q \cdot R . \Leftrightarrow a^{3} \equiv 4 b^{2} \text { or } 8 b^{2} & (\bmod .11) \\
13=P \cdot Q \cdot R . \Leftrightarrow a^{3} \equiv 7 b^{2} \text { or } 10 b^{2} & (\bmod 13) .
\end{array}
$$

For the proof of Theorem 1 we need the following lemmas.

LeMma 1 (DedeKIND [2, §2]). Let $g(X) \in \mathbf{Z}[X]$ be an irreducible polynomial, $\omega$ a root of $g(X)$ and $L=\mathbf{Q}(\omega)$. Let $p \in \mathbf{Z}$ be a prime and let

$$
g(X) \equiv \varphi_{1}(X)^{e_{1}} \cdots \varphi_{r}(X)^{e_{r}} \quad(\bmod . p)
$$

be the factorization of $g(X)$ into irreducible factors (mod. $p)$. If $p \nmid i(\omega)$, then $p=$ $P_{1}^{e_{1}} \cdots P_{r}^{e_{r}}$ in $L$; and for all $i, f\left(P_{i} / p\right)=\operatorname{deg}\left(\varphi_{i}(X)\right)$ and $P_{i}=\left(p, \varphi_{i}(\omega)\right)$.

LEMMA 2 (DEDEKIND [2, §3]). With the same notations of Lemma 1 , let

$$
g(X)=\varphi_{1}(X)^{e_{1}} \cdots \varphi_{r}(X)^{e_{r}}+p \cdot M(X), \quad M(X) \in \mathbf{Z}[X] .
$$

Then, $p \nmid i(\omega)$ is equivalent to $\varphi_{i}(X) \nmid M(X)(\bmod . p)$, for every $i$ such that $e_{i}>1$.

Lemma 3 (BAuer [1]). Let $g(X), \omega, L, p$, be as in Lemma 1. For every prime $P$ of $L$ lying over $p$, the quotient $v_{P}(\omega) / e(P / p)$ is equal to the slope of one of the sides of Newton's polygon of $g(X)$ with respect to $p$ and conversely, if $\lambda \in \mathbf{Q}$ is the slope of one side of that polygon, there exists a prime $P$ of $L$ lying over $p$ such that $v_{p}(\omega) / e(P / p)$ $=\lambda$.

The following lemma is an obvious consequence, in the cubic case, of more general results of Dedekind and Hensel (see [7, Theorem 4.8 and Proposition 6.10]).

LEMMA 4. With the same notations of Theorem 1 :

If $p=P \cdot Q^{2}$ in $K$ and $p \neq 2$, then $v_{p}(D)=1$.

If $p=P^{3}$ in $K$ and $p \neq 3$, then $v_{p}(D)=2$.

If $2=P \cdot Q^{2}$ in $K$, then $v_{2}(D)=2$ or 3 .

If $3=P^{3}$ in $K$, then $v_{3}(D)=3,4$ or 5 .

We also need the following easy result. 
LEMMA 5. With the same notations of Theorem 1:

If $p=P$ or $p=P \cdot Q \cdot R$ in $K$, then $\Delta \in \mathbf{Q}_{p}^{2}$.

If $p=P \cdot Q$ or $p=P \cdot Q^{2}$ in $K$, then $\Delta \notin \mathbf{Q}_{p}^{2}$.

\section{Proof of Theorem 1.}

Case I. $p|a, p| b$. By hypothesis $2 v_{p}(b) \neq 3 v_{p}(a)$, hence, the only possibilities are $0<2 v_{p}(b)<3 v_{p}(a)$ and $0<3 v_{p}(a)<2 v_{p}(b)$. In the first case, $v_{p}(b)=1$ or 2 and $v_{p}(a) \geqslant v_{p}(b)$, so that Newton's polygon of $f(X)$ with respect to $p$ has only one side with slope $\frac{1}{3}$ or $\frac{2}{3}$, hence, by Lemma 3, $p=P^{3}$. In the second case, $1=v_{p}(a)<v_{p}(b)$ and the polygon has two sides, one of them with slope $\frac{1}{2}$; again by Lemma 3 , we have $p=P \cdot Q^{2}$.

Case II. $p \nmid a, p \mid b$. In this case, by Lemma 3 there are at least two primes of $K$ over $p$. If $p \neq 2$, then $s_{p}=0$ and by Lemma $1, p=P \cdot Q \cdot R$ or $p=P \cdot Q$ according to $(a / p)=1$ or -1 .

If $p=2$, then $s_{2}>0$. If $s_{2}$ is odd, then 2 is ramified, so that $2=P \cdot Q^{2}$. Let us assume that $s_{2}$ is even. Now, by Lemma $5,2=P \cdot Q \cdot R$ if and only if $\Delta$ is a square in $\mathbf{Q}_{2}$, that is, if and only if $\Delta_{2} \equiv 1(\bmod .8)$. Finally, if $\Delta_{2} Z 1(\bmod .8)$, we have $f(X)=g(X) \cdot h(X)$ in $\mathbf{Q}_{2}[X]$, with $\operatorname{deg}(g(X))=1, \operatorname{deg}(h(X))=2$. both irreducible in $\mathbf{Q}_{2}[X]$. We have $2=P \cdot Q$ if and only if the quadratic extension of $\mathbf{Q}_{2}$ which define the roots of $h(X)$ is nonramified and that happens if and only if $\Delta_{2} \equiv 5$ (mod. 8) since $\mathbf{Q}_{2}(\sqrt{5})$ is the only nonramified quadratic extension of $\mathbf{Q}_{2}$ [7, p. 215].

Case III. $p \mid a, p \nmid b$. If $p \neq 3$, then $s_{p}=0$. Hence, by Lemma 1 the decomposition of $p$ depends only on the factorization of $f(X) \equiv X^{3}+b(\bmod . p)$. If $p \equiv-1$ $(\bmod .3), f(X)$ has only one root $(\bmod . p)$, hence $p=P \cdot Q$. If $p \equiv 1(\bmod .3), f(X)$ has three roots (mod. $p$ ) or none according to $b$ being a cubic residue (mod. $p$ ) or not.

For $p=3, f(X) \equiv(X+b)^{3}(\bmod .3)$. Making the substitution $\theta_{1}=\theta+b$ we obtain the equation

$$
f_{1}(X)=X^{3}-3 b X^{2}+\left(3 b^{2}-a\right) X-\left(b^{3}-a b-b\right) \text {. }
$$

If $a \neq 3(\bmod .9)$, we have $3 b^{2} \equiv 3 \neq a(\bmod .9)$ and Newton's polygon of $f_{1}(X)$ with respect to 3 gives a complete answer to our problem. In fact, if $b^{2} \equiv a+$ 1 (mod.9), then $3=P \cdot Q^{2}$ since the polygon has two sides, one of them with slope $\frac{1}{2}$; and if $b^{2} \neq a+1(\bmod .9)$, then $3=P^{3}$ since the polygon has only one side with slope $\frac{1}{3}$.

Assume now that $a \equiv 3(\bmod .9)$. If $b^{2} Z a+1(\bmod .27)$, then $3=P^{3}$ since the polygon has only one side with slope $\frac{1}{3}$ or $\frac{2}{3}$. If $b^{2} \equiv a+1(\bmod .27)$, making the substitution $\theta_{2}=\theta_{1} / 3$ we obtain the equation

$$
f_{2}(X)=X^{3}-b X^{2}+\frac{3 b^{2}-a}{9} X-\frac{b^{3}-a b-b}{27} .
$$

Observe first that the congruence $f_{2}(X) \equiv(X+c)^{3}(\bmod .3), c \in \mathbf{Z}$, is not possible. hence, $3 \neq P^{3}$, and $3=P$ if and only if $f_{2}(X)$ is irreducible (mod. 3$)$. Since $3 \neq P^{3}$, by Lemma 4 we have $3=P \cdot Q^{2}$ if and only if $s_{3}$ is odd; and by Lemma $5,3=P \cdot Q$ if and only if $s_{3}$ is even and $\Delta_{3} \equiv-1(\bmod .3)$. 
Finally, suppose that $s_{3}$ is even and $\Delta_{3} \equiv 1(\bmod .3)$ and let $D_{2}=\Delta / 27^{2}$ be the discriminant of $f_{2}(X)$. Under our assumptions $f_{2}(X)$ cannot have only one root (mod. 3) and since $b \neq 0(\bmod .3)$ it cannot have three simple roots (mod. 3), hence,

$$
s_{3}=6 \Leftrightarrow 3+D_{2} \Leftrightarrow f_{2}(X) \text { irreducible }(\bmod .3) \Leftrightarrow 3=P .
$$

Case IV. $p \nmid a b$. If $p=2$ or 3 , then $s_{p}=0$ and the assertions of Theorem 1 are an obvious consequence of Lemma 1. Let $p>3$. By Lemma 4 we have $p=P \cdot Q^{2}$ if and only if $s_{p}$ is odd. Moreover, since the congruence

$$
f(X) \equiv(X+c)^{3} \quad(\bmod . p), \quad c \in \mathbf{Z},
$$

is not possible, we can assure that $p \neq P^{3}$. Hence, by Lemma 4 we have $p=P \cdot Q$ if and only if $s_{p}$ is even and $\left(\Delta_{p} / p\right)=-1$. If $s_{p}$ is even and $\left(\Delta_{p} / p\right)=1$, then $p=P$ or $P \cdot Q \cdot R$ according to $f(X)$ being irreducible (mod. $p$ ) or not.

This ends the proof of Theorem 1.

We now apply Theorem 1 to obtain a direct computation of $D$ in terms of $a$ and $b$.

THEOREM 2. With the same notations, for every prime $p>3$ we have

$$
\begin{array}{lll}
v_{p}(D)=2 & \Leftrightarrow & 1 \leqslant v_{p}(b) \leqslant v_{p}(a), \\
v_{p}(D)=1 & \Leftrightarrow & s_{p} \text { odd, } \\
v_{p}(D)=0
\end{array} \quad \text { otherwise; }
$$

for $p=2$ we have

$$
\begin{aligned}
& v_{2}(D)=3 \quad \Leftrightarrow \quad \begin{array}{l}
s_{2} \text { odd }, \\
v_{2}(D)=2
\end{array} \\
& v_{2}(D)=0 \quad\left\{\begin{array}{l}
1 \leqslant v_{2}(b) \leqslant v_{2}(a), \text { or } \\
s_{2} \text { even and } \Delta_{2} \equiv 3(\bmod .4),
\end{array}\right.
\end{aligned}
$$

and for $p=3$ we have

$$
\begin{aligned}
& v_{3}(D)=5 \Leftrightarrow 1 \leqslant v_{3}(b)<v_{3}(a), \\
& v_{3}(D)=4 \Leftrightarrow\left\{\begin{array}{l}
v_{3}(a)=v_{3}(b)=2, \text { or } \\
a \equiv 3(\bmod .9), 3 \nmid b \text { and } b^{2} \geq 4(\bmod .9),
\end{array}\right. \\
& v_{3}(D)=3 \Leftrightarrow\left\{\begin{array}{l}
v_{3}(a)=v_{3}(b)=1, \text { or } \\
3 \mid a, 3 \nmid b, a \neq 3(\bmod .9) \text { and } b^{2} \neq a+1(\bmod .9), \text { or } \\
a \equiv 3(\bmod .9), b^{2} \equiv 4(\bmod .9) \text { and } b^{2} \neq a+1(\bmod .27),
\end{array}\right. \\
& v_{3}(D)=1 \Leftrightarrow\left\{\begin{array}{l}
1=v_{3}(a)<v_{3}(b), \text { or } \\
3 \mid a, a \geq 3(\bmod .9) \text { and } b^{2} \equiv a+1(\bmod .9), \text { or } \\
a \equiv 3(\bmod .9), b^{2} \equiv a+1(\bmod .27) \text { and } s_{3} \text { odd },
\end{array}\right. \\
& v_{3}(D)=0 \Leftrightarrow\left\{\begin{array}{l}
3 \nmid a, \text { or } \\
a \equiv 3(\bmod .9), b^{2} \equiv a+1(\bmod .27) \text { and } s_{3} \text { even } .
\end{array}\right.
\end{aligned}
$$


Proof. We prove only the results concerning $v_{3}(D) \geqslant 3$, which correspond to $3=P^{3}$, since all the other cases are an easy consequence of Theorem 1 and Lemma 4.

Since $v_{3}(D)=3$ if $v_{3}(\Delta)=3$ and $v_{3}(D)=4$ if $v_{3}(\Delta)$ is even, the computation of $v_{3}(\Delta)$ solves all the cases of $3=P^{3}$ except for the following:

(i) $1=v_{3}(b)<v_{3}(a)$,

(ii) $2=v_{3}(b)<v_{3}(a)$,

(iii) $a \equiv 3(\bmod .9), b^{2} \equiv 4(\bmod .9)$ and $b^{2} z a+1(\bmod .27)$.

In case (i), $v_{3}(\Delta)=5$ and by Lemma 2 we have $v_{3}(D)=5$ because $3+i(\theta)$. In case (ii) we have $v_{3}(\Delta)=7$. The element $\theta^{\prime}=\theta^{2} / 3$ is an integer and its irreducible polynomial is $X^{3}-(2 a / 3) X^{2}+\left(a^{2} / 9\right) X-\cdot\left(b^{2} / 27\right)$. By Lemma 2 we have $3+i\left(\theta^{\prime}\right)$ and it is easy to see that $i(\theta)=3 \cdot i\left(\theta^{\prime}\right)$, hence $v_{3}(D)=5$. In case (iii) we have $v_{3}(\Delta)=5$ and by Lemma $2,3 \mid i(\theta)$, so that $v_{3}(D)=3$.

REMARK. Clearly, this theorem enables us to compute at once $i(\theta)=\sqrt{(\Delta / D)}$ in terms of $a$ and $b$. That shortens considerably the computation of a basis of the integers of $K$ following the method of Voronoi (see [3, §17]).

Theorem 2 provides also an elementary proof of the following theorem, which was proved first by Hasse [5] and later by Martinet and Payan [6] using more elaborate arguments.

THEOREM 3 (HASSE). If $d$ denotes the discriminant of $\mathbf{Q}(\sqrt{D})$ we have

$$
D=d \cdot 3^{2 m} \cdot f^{2},
$$

where,

(i) $f=p_{1} \cdots p_{g}$ is a square-free integer, $(f, 3 d)=1$ and $p_{i} \equiv\left(d / p_{\imath}\right)(\bmod .3)$ for all $i$.

(ii) $0 \leqslant m \leqslant 2$; if $3 \nmid d$, then $m \neq 1$ and if $d \equiv 3(\bmod .9)$, then $m \neq 2$.

Proof. Let $D=d_{0} \cdot 3^{2 m} \cdot f_{0}^{2}, d_{0}$ square-free and $3 \nmid f_{0}$. If $d_{0} \equiv 1(\bmod .4)$, we have $d=d_{0}$. If $d_{0} \neq 1(\bmod .4)$, then $D Z 1(\bmod .4)$, hence, by Stickelberger's criterion (see $[7$, Theorem 2.5$])$ we have $D \equiv 0(\bmod .4)$, so that $2 \mid f_{0}$ and $d=4 d_{0}$ divides $D$. In all cases we can write $D$ as in (2).

By Lemma $4 f$ is square-free and $(f, 3 d)=1$. Let $p \neq 3$; we find

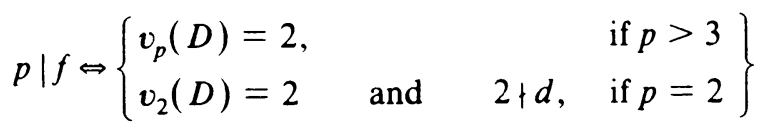

$$
\begin{aligned}
& \stackrel{\text { (Theorem 2) }}{\Leftrightarrow} 1 \leqslant v_{p}(b) \leqslant v_{p}(a) \stackrel{\text { (Theorem 1) }}{\Leftrightarrow} p=P^{3} .
\end{aligned}
$$

Therefore, if $p \mid f$ and $p>3$, we have $(d / p)=\left(\Delta_{p} / p\right)=(-3 / p) \equiv p(\bmod .3)$. And if $2 \mid f$, then $d \equiv \Delta_{2} \equiv 5(\bmod .8)$, so that $(d / 2)=-1 \equiv 2(\bmod .3)$. That ends the proof of (i).

By Lemma $4,0 \leqslant m \leqslant 2$ and $m \neq 1$ if $3 \nmid d$. If $3 \mid d$, then $(d / 3) \equiv \Delta_{3}(\bmod .3)$. Now, if $3 \mid d$ and $m=2$, by Theorem 2 we have $1 \leqslant v_{3}(b)<v_{3}(a)$ and in this case, always $\Delta_{3} \equiv-1(\bmod .3)$, so that $d \equiv 6(\bmod .9)$. That ends the proof of (ii). 
Finally, we find a characterization of the cubic fields in which 2 is a common index divisor. We recall that the index $i(L)$ of any number field $L$ is defined as

$$
i(L)=\text { g.c.d. }\{i(\omega) \mid \omega \in L \text { is an integer such that } L=\mathbf{Q}(\omega)\} \text {. }
$$

It is well known that if $K$ is a cubic field, then $i(K)=1$ or 2 and that $i(K)=2$ if and only if $2=P \cdot Q \cdot R$ in $K$ [4]. Therefore, our Theorem 1 furnishes the following simple criterion.

THEOREM 4. With the same notations of Theorem 1 ,

$$
i(K)=2 \Leftrightarrow a \text { odd }, b \text { even, } s_{2} \text { even and } \Delta_{2} \equiv 1(\bmod .8) \text {. }
$$

\section{REFERENCES}

1. M. Bauer, Zur allgemeinen Theorie der algebraischen Grössen, J. Reinc Angew. Math. 132 (1907), 21-32.

2. R. Dedekind, Üher den Zusammenhang zwischen der Theorie der Ideale und der höheren Kongruenzen. Abh. Kgl. Ges. Wiss. Göttingen 23 (1878), 1-23.

3. B. N. Delone and D. K. Faddeev, The theory of irrationalities of the third degree, Transl. Math. Monographs, vol. 10. Amer. Math. Soc., Providence, R. I., 1964.

4. H. T. Engstrom. On the common index divisors of an algehraic field. Trans. Amer. Math. Soc. 32 (1930), 223-237.

5. H. Hasse. Aritmetische Theorie der kubischen Zahlkörper auf klassenkörpertheoretischer (irundlage. Math. Z. 31 (1930), 565-582.

6. J. Martinet and J. J. Payan. Sur les extensions cubiques non-Galoisiennes des rationnels et leur cloture Galoisienne, J. Reine Angew. Math. 228 (1967), 15-37.

7. W. Narkiewicz. Elementary and analvtic theory of algebraic numbers, Monogr. Mat. 57 (1974).

8. L. Tornheim. Minimal hasis and inessential discriminant divisors for a cubic field, Pacific J. Math. 5 (1955), 623-631.

Seccio de Matematiques, Universitat autonoma de Barcelona, Bellaterra, Barcelona, Catalunya, Espanya

Postgrado de Ingenieria (P.E.A.M.), Universidad del Zulia, Maracaibo, Venezuela 